\title{
Recommandations canadiennes pour les pratiques optimales de soins de l'AVC, septième édition : l'acide acétylsalicylique pour la prévention d'événements vasculaires
}

\author{
Theodore Wein MD, M. Patrice Lindsay RN PhD, David J. Gladstone MD PhD, Alexandre Poppe MD CM, \\ Alan Bell MD, Leanne K. Casaubon MD MSc, Norine Foley MSc, Shelagh B. Coutts MD MBChB, Jafna Cox MD, \\ James Douketis MD, Thalia Field MD MHSc, Laura Gioia MD MSc, Jeffrey Habert MD, Eddy Lang MD MDCM, \\ Shamir R. Mehta MD MSc, Christine Papoushek PharmD, William Semchuk PharmD MSc, Mikul Sharma MD MSc, \\ Jacob A. Udell MD MPH, Stephanie Lawrence BA Dip. J., Anita Mountain MD, Gord Gubitz MD, \\ Dar Dowlatshahi MD PhD, Anne Simard MHSc BJ, Andrea de Jong RN MN, Eric E. Smith MD MPH; \\ pour la Fondation des maladies du cœur et de l'AVC du Canada (Cœur + AVC), en collaboration avec le \\ Consortium Neurovasculaire Canadien
}

Citation: CMAJ 2020 March 23;192:E302-11. doi: 10.1503/cmaj.191599-f

Baladodiffusions CMAJ : entrevue en anglais avec l'auteur à : https://soundcloud.com/cmajpodcasts/191599-guide; entrevue en français au https://soundcloud.com/cmajpodcasts/191599-guide-fre

n 2016, au Canada (à l'exclusion du Québec), 270204 personnes ont été admises à l'hôpital pour un trouble cardiaque, un accident vasculaire cérébral (AVC) ou un déficit cognitif d'origine vasculaire; de ce nombre, 107391 étaient des femmes, 162813 étaient des hommes et 91524 sont décédés ${ }^{1}$. Cela équivaut à 1 décès sur 3 au Canada, loin devant d'autres maladies; les maladies du cœur, l'AVC et les déficits cognitifs d'origine vasculaire causent $13 \%$ plus de décès que tous les types de cancers réunis ${ }^{1}$.

Les bienfaits de l'acide acétylsalicylique (AAS) en prévention secondaire de la maladie cardiovasculaire athéroscléreuse sont bien connus. En revanche, même si le traitement avec l'AAS à faible dose a déjà été fréquemment recommandé en prévention primaire de la maladie cardiovasculaire athéroscléreuse, cette pratique est à présent remise en question à la lumière de données récentes. Trois grands essais randomisés et contrôlés sur la prévention primaire n'ont fait état d'aucun bienfait lié à l'AAS pour la prévention des événements ou de la mortalité cardiovasculaires chez les adultes âgés en bonne santé ( $>65$ ans selon l'étude ASPREE [Aspirin in Reducing Events in the Elderly]) ou chez les personnes atteintes de diabète ou présentant d'autres facteurs de risque $e^{2-4}$. De plus, 2 revues systématiques récentes qui ont inclus les conclusions de ces essais ont fait état de résultats quelque peu contradictoires ${ }^{5,6}$. Elles ont toutes les deux montré que le risque de saignement majeur augmentait de manière importante chez les participants recevant l'AAS. Une des

\section{POINTS CLÉS}

- L'acide acétylsalicylique demeure fortement indiqué en prévention secondaire chez les patients atteints de maladie cardiovasculaire, vasculaire cérébrale ou artérielle périphérique évidente.

- L'acide acétylsalicylique (AAS) n'est plus recommandé en prévention primaire chez les personnes ne présentant aucun antécédent de maladie cardiovasculaire, d'accident vasculaire cérébral (AVC) ou de maladie artérielle périphérique symptomatiques; les risques associés à son utilisation quotidienne en surpassent potentiellement les bienfaits.

- La révision de ces recommandations constitue une occasion d'accroître nos efforts de prévention primaire par l'adoption de saines habitudes de vie, par des modifications au mode de vie et la prise en charge des facteurs de risque cardiovasculaires.

- La décision d'instaurer, de cesser ou de maintenir un traitement par AAS doit être individualisée, et devrait se faire après un processus de décision partagée entre les professionnels de la santé et leurs patients, en tenant compte des risques et des bienfaits, mais aussi des valeurs et des préférences.

- D'importantes questions demeurent, notamment en ce qui concerne les bienfaits de l'AAS chez les patients plus jeunes exposés à un risque élevé, les personnes qui présentent une maladie vasculaire sous-clinique et une athérosclérose asymptomatique, et les conséquences de la cessation de l'AAS chez les patients qui l'utilisent en prévention primaire depuis longtemps. 
2 revues systématiques a toutefois révélé que l'utilisation d'AAS ne réduisait pas le risque de mortalité, toutes causes confondues, ou d'AVC ischémique ${ }^{6}$, tandis que l'autre a établi un lien entre l'AAS et la réduction du risque pour un éventail mixte composé de mortalité cardiovasculaire, infarctus du myocarde non fatal, AVC non fatal et AVC ischémique ${ }^{5}$.

Étant donné une meilleure connaissance des risques et bienfaits de l'AAS, de la baisse globale des taux d'événements cardiovasculaires dans les populations actuelles comparativement aux populations des études antérieures, et des recommandations contradictoires des lignes directrices et des revues systématiques, les médecins se questionnent de plus en plus au sujet du rôle de l'AAS en prévention primaire ${ }^{2}$. On ignore le nombre réel de Canadiens et de Canadiennes qui prennent de l'AAS en prophylaxie (sur ordonnance, ou de leur propre chef), mais il est probablement substantiel. Selon une étude américaine, les résultats d'un sondage mené auprès de la population ont montré que 29 millions de personnes aux États-Unis prenaient de l'AAS, dont $23 \%$ sans recommandation médicale ${ }^{7}$.

Cette série de recommandations vise à orienter l'utilisation de l'AAS en prévention primaire des événements vasculaires. Les lignes directrices abordent en outre la prévention secondaire de I'AVC afin de mettre en évidence les différences entre ces 2 domaines cliniques. La recommandation concernant l'utilisation de l'AAS en prévention primaire des événements vasculaires est un nouvel ajout à l'éventail des Recommandations canadiennes pour les pratiques optimales de soins de l'AVC de la Fondation des maladies du cœur et de l'AVC du Canada (" Cœur + AVC »). La recommandation concernant la prévention secondaire de l'AVC provient d'une série de recommandations existante à cet effet, qui a été mise à jour simultanément.

Données probantes à l'appui, ces recommandations répondent au besoin d'orientation des professionnels de la santé et du public, et elles permettent l'harmonisation et l'uniformité avec la position d'autres organismes canadiens, tels que Thrombose Canada et la Société canadienne de cardiologie, qui ont aussi publié des recommandations et des conseils cliniques au sujet de l'utilisation de l'AAS en prévention primaire ${ }^{8,9}$. Les objectifs qui sous-tendent la préparation et la diffusion de ces recommandations ainsi que leur application par les professionnels de la santé et le public sont d'optimiser les soins fondés sur des données probantes partout au Canada, d'harmoniser les pratiques, et de combler le fossé qui sépare la science actuelle et la pratique clinique.

\section{Portée}

Les Recommandations canadiennes pour les pratiques optimales de soins de l'AVC visent à fournir des lignes directrices à jour fondées sur des données probantes pour la prévention et la prise en charge des maladies qui préoccupent Cœur + AVC (soit l'AVC, l'accident ischémique transitoire [AIT] et les déficits cognitifs d'origine vasculaire, en plus des maladies du cœur, ainsi que leurs répercussions sur les personnes atteintes). Elles visent aussi à promouvoir un rétablissement optimal et la réinsertion sociale de quiconque a été touché directement ou indirectement (c.-à-d., les patients, les familles et les aidants).
Cet ensemble de recommandations est unique en ce qui concerne les Recommandations canadiennes pour les pratiques optimales de soins de l'AVC en ce sens qu'il ne porte pas uniquement sur l'AVC; en effet, il s'agit de la prévention primaire d'un éventail de maladies, y compris les maladies cardiovasculaires, les maladies vasculaires cérébrales, les déficits cognitifs d'origine vasculaire et les maladies artérielles périphériques. Ces lignes directrices incluent des recommandations concernant l'utilisation de l'AAS en prévention primaire et secondaire. Les recommandations sur la prévention secondaire incluses dans ces lignes directrices se limitent exclusivement à l'AAS en monothérapie et ne portent pas sur le choix plus vaste des agents antiplaquettaires actuellement offerts pour cette indication. Les recommandations sur la prévention secondaire au moyen de ces agents se trouvent dans les lignes directrices sur la prévention secondaire de l'AVC des Recommandations canadiennes pour les pratiques optimales de soins de l'AVC, lesquelles ont été mises à jour en même temps que cette ligne directrice sur l'AAS, et au moyen de la même méthodologie rigoureuse ${ }^{10}$.

Ces recommandations s'adressent à tous les professionnels de la santé pour l'ensemble du continuum de soins; aux gens qui ont subi un AVC ou sont atteints de maladie du cœur, de maladie vasculaire ou de déficit cognitif d'origine vasculaire; ainsi qu'au grand public. Les responsables des orientations politiques, les planificateurs, les organismes subventionnaires, les cadres supérieurs et les administrateurs des systèmes de santé responsables de la coordination et de la prestation des services de santé à l'échelle d'une province ou d'une région pourraient aussi trouver ce document pertinent et applicable à leur travail.

\section{Recommandations}

Trois recommandations ont été formulées au sujet de l'utilisation de l'AAS en prévention des événements vasculaires (encadré no 1). La première réitère l'importance du rôle de l'AAS en prévention secondaire chez les personnes qui ont déjà subi un premier événement vasculaire. La seconde découle directement des nouvelles données probantes à l'encontre de l'utilisation de l'AAS chez les personnes en bonne santé qui n'ont aucun antécédent d'événement vasculaire. La troisième rappelle l'importance d'une prise de décision partagée entre le médecin et le patient pour s'assurer que les valeurs et préférences de ce dernier sont prises en compte. Pour de plus amples renseignements sur les données probantes à l'appui de ces recommandations, on peut consulter l'annexe 1 (www.cmaj.ca/lookup/suppl/doi:10.1503/ cmaj.191599/-/DC2) et le document intégral des lignes directrices (https://www.pratiquesoptimalesavc.ca/recommandations/AAS -par-prevention).

\section{Prévention secondaire}

L'acide acétylsalicylique est fortement recommandé en prévention secondaire chez les personnes atteintes de maladie cardiovasculaire, vasculaire cérébrale ou artérielle périphérique symptomatique (niveau de preuve $A)^{11-13}$. 
Encadré no 1 : Recommandations concernant l'utilisation de l'acide acétylsalicylique en prévention des événements vasculaires*

\section{Prévention secondaire $\dagger$}

L'acide acétylsalicylique (AAS) est fortement recommandé en prévention secondaire chez les individus atteints de maladie cardiovasculaire, vasculaire cérébrale, ou artérielle périphérique symptomatique (niveau de preuve $\mathrm{A})^{11-13}$.

\section{Prévention primaire}

L'utilisation de l'AAS n'est pas recommandée en prévention primaire d'un premier événement vasculaire (niveau de preuve A) ${ }^{2,4-6}$.

- Cette recommandation concerne les individus présentant des facteurs de risque vasculaire et n'ayant subi aucun événement vasculaire (niveau de preuve $A$ ) ${ }^{2,4,6}$, et les individus âgés en bonne santé ne présentant pas de facteurs de risque vasculaire (niveau de preuve $B)^{3}$.

- Les bienfaits réels de l'AAS chez les individus atteints d'athérosclérose asymptomatique sont incertains (niveau de preuve $B)^{14,15}$

\section{Prise de décision partagée}

Les professionnels de la santé (médecins de première ligne ou spécialistes, infirmiers et infirmiers praticiens, pharmaciens, adjoints médicaux) devraient engager le dialogue avec les patients et leurs aidants au sujet de l'utilisation de l'AAS en prévention primaire de la maladie vasculaire. Il faut tenir compte, au cas par cas, des risques, des bienfaits, des valeurs et des préférences de chacun pour une prise de décision partagée éclairée concernant l'instauration, la cessation ou le maintien du traitement par l'AAS pour la prévention primaire de la maladie vasculaire (niveau de preuve $B)^{16,17}$.

*Consultez l'annexe 2 pour les tableaux afférents aux données probantes comparant les paramètres des essais cliniques randomisés et des revues systématiques. †Pour plus de renseignements sur l'utilisation de l'AAS et d'autres antiplaquettaires en prévention secondaire, consultez les Recommandations canadiennes pour les pratiques optimales de soins de l'AVC, module sur la prévention secondaire de I'AVC ${ }^{10}$, les Lignes directrices canadiennes relatives au traitement antiplaquettaire et l'utilisation des anticoagulants ${ }^{9}$ de la Société canadienne de cardiologie et les guides cliniques de l'organisme Thrombose Canada ${ }^{8}$.

Les bienfaits de l'AAS en prévention secondaire sont bien connus. La prise quotidienne d'AAS à faible dose réduit le risque d'événements vasculaires, y compris l'infarctus du myocarde, l'AVC et les décès d'origine vasculaire chez les patients qui ont déjà subi un événement vasculaire. L'Antithrombotic Trialists' Collaboration (2002) a procédé à une méta-analyse des données provenant de plusieurs essais volumineux montrant la constance des effets de l'AAS sur la prévention des récurrences d'événements cardiovasculaires ${ }^{11}$. Par exemple, comparativement au placebo, l'AAS a permis de réduire de 36 le nombre d'événements vasculaires graves (écart type [É.-T.] 5) par 1000 patients/année chez des patients ayant déjà subi un infarctus du myocarde et également de 36 (É.-T. 6) par 1000 patients/année chez ceux qui avaient des antécédents d'AVC ou d'AIT. Chez les patients atteints d'une maladie artérielle périphérique, comparativement au placebo, l'AAS a permis de réduire de $23 \%$ le risque d'événements vasculaires graves (É.-T. 8\%). Des réductions similaires du risque ont aussi été observées chez des patients qui présentaient des problèmes d'angine de poitrine stable ou instable ${ }^{11}$. Pour de plus amples renseignements sur les données probantes à l'appui de cette recommandation, on peut consulter https://www. pratiquesoptimalesavc.ca/recommandations/AAS-par-prevention.

\section{Prévention primaire}

L'utilisation de l'acide acétylsalicylique n'est pas recommandée en prévention primaire d'un premier événement vasculaire (niveau de preuve $A)^{2,4,6}$.

- Cette recommandation concerne les individus présentant des facteurs de risque d'événements vasculaires qui n'ont encore subi aucun événement vasculaire (niveau de preuve $A$ )2,4,6 et les individus âgés en bonne santé ne présentant pas de facteurs de risque vasculaire (niveau de preuve $B)^{3}$.

- Les bienfaits réels de l'AAS chez les individus atteints d'athérosclérose asymptomatique sont incertains (niveau de preuve $B)^{14,15}$.

\section{Réduction des événements vasculaires}

Le corpus de données probantes utilisé pour formuler la recommandation concernant l'utilisation de l'AAS en prévention primaire provient principalement de 3 essais randomisés et contrôlés (ERC) publiés récemment. Chacun a comparé les bienfaits potentiels de 100 mg d'AAS à ceux d'un placebo chez des personnes sans maladie cardiovasculaire préexistante, ce qui était défini de manière légèrement différente dans les 3 essais (annexe 2; www. cmaj.ca/lookup/suppl/doi:10.1503/cmaj.191599/-/DC2).

Les 3 essais reposaient sur des échantillons volumineux (de > 12000 à > 19000 participants). L'étude ARRIVE² (Aspirin to Reduce Risk of Initial Vascular Events) incluait des hommes de 55 ans et plus présentant de 2 à 4 facteurs de risque de problèmes cardiovasculaires et des femmes de 60 ans et plus présentant 3 facteurs de risque ou plus, et excluait les personnes atteintes de diabète. L'étude ASPREE $^{3}$ incluait des hommes et des femmes australiens et américains de 70 ans et plus (ou de 65 ans et plus parmi les américains de race noir ou hispanique). L'étude ASCEND 4 (A Study of Cardiovascular Events in Diabetes) incluait des hommes et des femmes de 40 ans et plus atteints de diabète de type 1 ou de type 2. L'âge moyen des participants était de 64 ans dans les études ARRIVE² et ASCEND ${ }^{4}$, et de 74 ans dans l'étude ASPREE ${ }^{3}$. L'utilisation courante d'AAS ou d'anticoagulants était un critère d'exclusion pour ces 3 études; toutefois, $36 \%$ des participants de l'étude ASCEND ${ }^{4}$ avaient utilisé de l'AAS avant leur sélection, tandis que $11 \%$ des participants de l'essai ASPREE ${ }^{3}$ avaient utilisé de l'AAS précédemment. La durée médiane du suivi a varié de 4,7 à 7,1 ans.

Les résultats de 2 essais ont été négatifs (ARRIVE², $A S P R E E^{3}$ ), c'est-à-dire que le risque d'événement cardiovasculaire n'a pas considérablement diminué dans les groupes avec l'AAS. Dans les études $A R R I V E^{2}$ et $A S P R E E^{3}$, les risques relatifs associés à l'AAS pour le paramètre principal ont été de 0,96 (intervalle de confiance [IC] de $95 \%, 0,81-1,13$ ) et de 0,95 (IC de $95 \%, 0,83-1,08$ ), respectivement. Dans l'étude $\mathrm{ASCEND}^{4}$, le risque de survenue du paramètre principal (premier événement vasculaire grave [infarctus du myocarde, AVC, AIT ou décès d'origine cardiovasculaire]) a été considérablement moindre dans le groupe avec l'AAS (8,5\% c. 9,6\%; risque relatif [RR] 0,88, IC de $95 \%, 0,79-0,97$ ).

Les résultats de ces 3 essais ont été intégrés à 2 revues systématiques, dont les conclusions se sont révélées contradictoires ${ }^{5,6}$. L'utilisation d'AAS n'a pas réduit le risque de mortalité, toutes causes confondues, ni d'AVC ischémique ${ }^{6}$, mais a réduit le risque d'un résultat mixte composé de mortalité cardiovasculaire, 
infarctus du myocarde non fatal, AVC non fatal et AVC ischémique ${ }^{5}$. Chacune de ces revues incluait les résultats des mêmes 11 ERC, mais celle par Zheng et Roddick ${ }^{5}$ incluait 2 autres essais (POPADAD [Prevention of Progression of Arterial Disease and Diabetes] $2008^{18}$, et AAA [Aspirin for Asymptomatic Atherosclerosis] 2010 14 ). Dans 1 des 2 revues, les critères d'inclusion se limitaient aux participants n'ayant présenté aucune maladie cardiovasculaire ${ }^{5}$, et dans l'autre ils incluaient les individus ayant des antécédents d'athérosclérose ${ }^{6}$.

\section{Risques}

Dans les 3 essais susmentionnés (ASPREE 3 , ARRIVE ${ }^{2}$ et ASCEND), le risque de saignement majeur a considérablement augmenté avec le traitement par AAS (ASPREE : risque relatif [RR] 1,38, IC de $95 \%, 1,18-1,62$; ARRIVE [tout saignement digestif] : RR 2,11, IC de $95 \%, 1,36-3,28$; et ASCEND : RR 1,29, IC de $95 \%, 1,09-1,52$ ).

Dans une revue systématique et méta-analyse conçue spécifiquement pour mesurer le risque d'hémorragie intracrânienne associé à l'utilisation d'AAS, Huang et ses collaborateurs ${ }^{19}$ ont inclus les résultats de 13 ERC $(n=134446)$ regroupant des personnes sans maladies du cœur symptomatiques préexistantes (p. ex., coronaropathie, AVC ou maladie artérielle périphérique). Les essais ont comparé l'AAS à faible dose ( $\leq 100 \mathrm{mg} / \mathrm{j} \geq 6$ mois) à un placebo ou à l'absence de traitement et ont mesuré exclusivement les résultats sur le plan des hémorragies intracrâniennes. L'utilisation d'AAS a été associée à une augmentation importante du risque de tout saignement intracrânien (RR 1,37 , IC de $95 \%, 1,13-1,66 ; n=8$ essais; 2 hémorragies intracrâniennes supplémentaires par 1000 personnes). Selon une analyse de sensibilité excluant les résultats de l'étude ASPREE ${ }^{3}$, qui n'incluait que des personnes âgées (de $>65$ ans et $\geq 70$ ans selon la race), le risque devenait non significatif. L'AAS n'a pas été associé à une augmentation considérable du risque d'hémorragie intracérébrale ou sous-arachnoïdienne. Dans une analyse de sous-groupes, les participants d'origine asiatique et les personnes qui avaient un indice de masse corporelle $<25$ et prenaient de l'AAS ont été exposés à un risque considérablement plus élevé d'hémorragie intracérébrale. Le risque de saignement majeur a aussi augmenté selon les résultats de 2 autres méta-analyses récentes ${ }^{5,6}$.

\section{Zones d'incertitude}

En raison des données probantes insuffisantes quant à un effet bénéfique de l'AAS en prévention primaire des événements cardiovasculaires majeurs, rien ne justifie l'utilisation de l'AAS en prévention primaire chez les patients, peu importe le degré de risque, y compris un risque élevé. Toutefois, Marquis-Gravel et ses collaborateurs ${ }^{20}$ ont mis en lumière plusieurs zones d'incertitude qui persistent relativement à l'utilisation de l'AAS en prévention primaire, après la conclusion des essais ARRIVE, ASPREE et ASCEND. Des facteurs tels que le poids corporel et le sexe ont été identifiés comme facteurs potentiels de modification de l'effet. Dans une méta-analyse sur données agrégées des résultats individuels de patients provenant de $10 \mathrm{ERC}^{21}$, le risque d'événement cardiovasculaire associé à l'utilisation de 75-100 mg d'AAS en prévention primaire a été moindre chez les participants de poids élevé, tandis que l'AAS à faible dose a exercé un effet préventif plus marqué chez les participants qui pesaient de 50 à $69 \mathrm{~kg}$. Dans cette même étude, des doses de 350 et 500 mg d'AAS en prévention primaire ont été associées à une baisse du risque d'événement cardiovasculaire chez les participants pesant $\geq 70 \mathrm{~kg}$.

On a également étudié l'efficacité de l'AAS à réduire le risque d'événement cardiovasculaire, y compris l'AIT, l'AVC, l'infarctus du myocarde, l'angine de poitrine instable ou le décès chez les personnes atteints d'athérosclérose asymptomatique. Même si on a suggéré que l'AAS à faible dose puisse entraîner un changement de la composition de la plaque à l'intérieur des vaisseaux sanguins, l'utilisation quotidienne de $325 \mathrm{mg}$ d'AAS pendant 2 ans chez des personnes présentant une sténose carotidienne ( $\geq 50 \%)$ n'a été associée à aucune réduction des événements vasculaires comparativement au placebo ${ }^{15}$.

Le rôle du sexe comme facteur potentiel de modification de l'effet a été moins évident à cerner; en effet, nous n'avons relevé aucune interaction importante entre le sexe et l'efficacité de l'AAS dans les essais ARRIVE, ASCEND ou ASPREE. Dans une méta-analyse plus ancienne, qui incluait les résultats de $6 \mathrm{ERC}$, Berger et ses collaborateurs ${ }^{22}$ ont laissé entendre que l'AAS réduisait le risque d'infarctus du myocarde seulement chez les hommes, et le risque de tout AVC et d'AVC ischémique seulement chez les femmes. Le rôle du genre n'a été mentionné dans les résultats d'aucune de ces études.

\section{Prise de décision partagée}

Les professionnels de la santé (médecins de première ligne ou spécialistes, infirmiers et infirmiers praticiens, pharmaciens, adjoints médicaux) devraient engager le dialogue avec les patients et leurs aidants au sujet de l'utilisation de l'acide acétylsalicylique en prévention primaire de la maladie vasculaire. Il faut tenir compte, au cas par cas, des risques, des bienfaits, des valeurs et des préférences de chacun pour une prise de décision partagée éclairée concernant l'instauration, la cessation ou le maintien de l'acide acétylsalicylique pour la prévention primaire de la maladie vasculaire (niveau de preuve $B)^{16,17}$.

Même si l'utilisation à long terme de l'AAS n'est pas préconisée en prévention primaire d'un premier événement vasculaire, dans les recommandations, on reconnaît que la décision d'instaurer, de cesser ou de maintenir un traitement avec l'AAS mérite d'être prise au cas par cas, après une évaluation du rapport risquesbienfaits, et une discussion médecin-patient au sujet des risques et bienfaits possibles et des solutions de rechange. Ce processus de prise de décision partagée doit se fonder sur les données cliniques ainsi que sur les préférences et valeurs du patient une fois que celui-ci a été renseigné ${ }^{23,24}$.

Pour la plupart des patients, cesser l'AAS sera le meilleur choix. Toutefois, certains pourraient préférer continuer le traitement pour des raisons personnelles, par exemple : antécédents familiaux majeurs de maladie vasculaire, ou importance plus grande accordée à la prévention des événements vasculaires qu'à l'évitement des risques de saignement majeur. 
On ne dispose actuellement d'aucune donnée probante quant au rôle de la prise de décision partagée dans le contexte d'un traitement avec l'AAS en prévention primaire, quoique des interventions visant à appuyer la prise de décision partagée ont été évaluées pour le traitement de l'hypertension artérielle ${ }^{25,26}$, de la fibrillation auriculaire ${ }^{27}$ et du diabète ${ }^{28}$.

\section{Méthodologie}

\section{Composition du comité de rédaction des lignes directrices} Un comité interdisciplinaire d'experts chargé de la rédaction a été formé par Cœur + AVC pour faire une revue de la documentation et analyser l'utilité de l'AAS en prévention primaire des événements vasculaires. Le comité incluait des neurologues spécialistes de l'AVC, des cardiologues, des spécialistes en maladie vasculaire, des médecins de famille, un urgentologue, un physiatre, des pharmaciens, des infirmiers et un épidémiologiste. Les membres ont été sélectionnés après un appel de candidatures pour former les comités de rédaction des Recommandations canadiennes pour les pratiques optimales de soins de l'AVC en prévention primaire et secondaire. Le président du comité de rédaction (T.W.) et les membres du comité consultatif pour les Recommandations canadiennes pour les pratiques optimales de soins de l'AVC ont sélectionné les 18 membres du comité principal de rédaction en veillant à la représentativité géographique, urbaine et rurale, et interdisciplinaire, ainsi qu'à l'équilibre entre les genres, et les années d'expérience.

Dans le cadre de la méthodologie appliquée aux Recommandations canadiennes pour les pratiques optimales de soins de I'AVC, un comité communautaire de consultation et révision, composé de 8 patients et aidants, a pris part à l'examen et à la préparation de ces recommandations ${ }^{29}$ en collaboration avec le comité de rédaction.

Un groupe de 15 réviseurs externes n'ayant pas participé au processus de préparation des lignes directrices a été formé pour en faire la révision finale. Ce comité d'examen externe avait la même représentativité que le comité de rédaction. Les membres ont été choisis à partir de notre liste originale de personnes candidates qui n'avaient pas été retenues pour le comité de rédaction et au moyen d'une revue de la documentation afin de trouver des professionnels connaissant bien le sujet.

Ces recommandations ont été préparées en collaboration avec le Consortium Neurovasculaire Canadien, dont plusieurs des membres (T.W., D. J.G., A.P., L.K. C., S. B.C., T. F., L. G., M.S., G.G., D.D. et E.E.S.) ont fait partie du comité de rédaction. La Société canadienne de cardiologie comptait aussi 2 représentants officiels (J.C. et J.A. U.) parmi les membres du comité de rédaction.

\section{Préparation des lignes directrices}

Le processus de préparation et de mise à jour des Recommandations canadiennes pour les pratiques optimales de soins de l'AVC suit un cadre standardisé rigoureux, adapté de la Grille d'évaluation de la qualité des recommandations pour la pratique clinique $^{30,31}$ et tient compte de tous les critères définis dans le modèle de l'AGREE Research Trust (Appraisal of Guidelines for
Research and Evaluation) ${ }^{32}$. La méthodologie a été appliquée aux mises à jour précédentes des Recommandations canadiennes pour les pratiques optimales de soins de l'AVC et on peut la trouver sur le site Web des Pratiques optimales pour les soins de l'AVC au Canada, à l'adresse https://www.pratiquesoptimalesavc.ca/.

Du personnel chevronné a procédé à des interrogations de la documentation pour recenser les articles révisés par des pairs ayant porté sur l'utilisation de l'AAS en monothérapie en prévention des événements vasculaires et le rôle de la prise de décision partagée dans la réduction du risque de problèmes vasculaires. Nous avons inclus des revues systématiques, des méta-analyses, des essais randomisés et contrôlés et des études d'observation, selon leur disponibilité. La documentation consultée pour le présent module était à jour en date de novembre 2019. La stratégie d'interrogation est présentée à l'annexe 2.

À l'aide d'un modèle d'abstraction standardisé, des tableaux des données probantes, incluant le contenu des études sélectionnées, ont été dressés par le personnel chevronné; ces tableaux ont été remis au comité de rédaction pour consultation. Le comité de rédaction a mené des discussions et des débats au sujet de la solidité, de l'importance, de la pertinence clinique et de l'applicabilité des données probantes, et après consensus, a rédigé l'ébauche de la série de recommandations proposées. Pendant ce processus, nous avons identifié d'autres sources dans la documentation et nous en avons tenu compte au moment de finaliser les recommandations. On peut trouver la définition des termes utilisés dans les recommandations du présent document (p. ex., prévention primaire, prévention secondaire) à l'adresse suivante : https://www.pratiquesoptimalesavc.ca/recommandations /AAS-par-prevention.

Nous avons attribué un niveau de preuve, de $\mathrm{A}$ à $\mathrm{C}$, à chaque recommandation, indiquant la solidité des données probantes selon les critères définis dans l'encadré no 2 . Ces critères s'inspirent des travaux de Guyatt et collaborateurs ${ }^{33}$. En ce qui concerne la rédaction et l'inclusion des recommandations de " niveau C », nous avons obtenu un consensus du comité de rédaction et nous l'avons validé par un processus d'examen interne et externe. Nous avons utilisé ce niveau de preuve avec prudence, et uniquement lorsqu'il y a absence de données probantes solides dans les domaines en question, et si le comité de rédaction estimait qu'une certaine forme d'orientation devait être proposée.

Pendant la préparation des recommandations sur l'utilisation de l'AAS en prévention primaire, nous avons craint qu'il puisse y avoir une certaine confusion parmi le public et les professionnels de la santé au sujet de l'utilisation de l'AAS en prévention secondaire. La majorité des membres ont en effet pu citer plusieurs cas dans leurs pratiques cliniques où des patients ayant des antécédents d'AVC ou de coronaropathie avaient cessé l'AAS de leur propre chef, en raison de la couverture médiatique des nouveaux essais sur l'AAS en prévention primaire. C'est pourquoi nous avons décidé d'élargir la portée de ces lignes directrices afin d'y inclure une recommandation relative à la prévention secondaire. Cette recommandation a été préparée conjointement avec un autre comité de rédaction des lignes directrices qui a travaillé sur la prévention secondaire de 
Encadré no 2 : Description des niveaux de preuve afférents aux Recommandations canadiennes pour les pratiques optimales de soins de l'AVC ${ }^{33}$

Preuves tirées d'une méta-analyse d'ERC, ou résultats concordants de 2 ERC ou plus. Les effets désirables surpassent nettement les conséquences indésirables ou vice versa.

Dans leur formulation, les recommandations fondées sur un tel niveau de preuve incluent des expressions comme « forte recommandation »; telle ou telle mesure « devrait (ou ne devrait pas) être appliquée».

Preuves tirées d'un seul ERC ou résultats concordants de 2 essais non randomisés ou non contrôlés bien conçus et études d'observation volumineuses. Méta-analyses d'études non randomisées ou d'observation. Les effets désirables surpassent ou sont en équilibre étroit avec les conséquences indésirables, ou vice versa.

Dans leur formulation, les recommandations fondées sur un tel niveau de preuve incluent des expressions comme « est recommandé »; " devrait être envisagé »; et dans certains cas où le comité de rédaction a atteint un solide consensus, telle ou telle mesure « devrait (ou ne devrait pas) être appliquée chez la plupart des groupes ou chez des groupes spécifiques selon le cas ».

C Consensus du comité de rédaction sur les thématiques s'appuyant sur des résultats de recherche limités. Les effets désirables surpassent ou sont en équilibre étroit avec les conséquences indésirables, ou vice versa.

Dans leur formulation, les recommandations fondées sur un tel niveau de preuve incluent des expressions comme " peut être envisagé » ou « est raisonnable ».

Pour considération Conseil sur la pratique raisonnable fourni après consensus du comité de rédaction au sujet d'enjeux cliniques spécifiques clinique courants ou controversés et absence de données probantes tirées de la recherche pour orienter la pratique. Aucun niveau de preuve n'est assigné aux considérations cliniques.

Note: $\mathrm{AVC}=$ accident vasculaire cérébral, $\mathrm{ERC}=$ essai randomisé et contrôlé.

l'AVC, en suivant la démarche rigoureuse appliquée à la rédaction des Recommandations canadiennes pour les pratiques optimales de soins de l'AVC. Plusieurs membres du comité de rédaction concernant la prévention primaire ont fait partie des 2 groupes. La description complète de la méthodologie appliquée à la préparation des lignes directrices pour la prévention secondaire est accessible sur le site https://www. pratiquesoptimalesavc.ca/.

\section{Revue}

L'ébauche du document a fait l'objet d'un examen interne par le comité consultatif pour les Recommandations canadiennes pour les pratiques optimales de soins de l'AVC, avant d'être envoyée à un comité d'examen externe pour révision. Elle a aussi été envoyée au Consortium Neurovasculaire Canadien, à la Société canadienne de cardiologie, à l'organisme Thrombose Canada, à la Société canadienne des pharmaciens d'hôpitaux et à l'Association des pharmaciens du Canada, pour qu'ils l'examinent et l'approuvent. Nous avons soigneusement tenu compte de tous les commentaires formulés durant le processus de révision et nous les avons intégrés à la version finale, le cas échéant.

Les lignes directrices bénéficient de l'appui du Consortium Neurovasculaire Canadien, de la Société canadienne de cardiologie, de l'organisme Thrombose Canada, de la Société canadienne des pharmaciens d'hôpitaux, de l'Association des pharmaciens du Canada et de la Nurse Practitioners' Association of Ontario.

On trouvera plus d'information sur la méthodologie appliquée à la rédaction des lignes directrices dans le document intégral, accessible à l'adresse https://www.pratiquesoptimalesavc. $\mathrm{ca} /$ recommandations/AAS-par-prevention. Nous mettrons ces lignes directrices à jour régulièrement, soit tous les 2 ou 3 ans, afin d'y intégrer les données probantes émergentes.

\section{Gestion des intérêts concurrents}

Tous les participants potentiels au processus de préparation et de révision des recommandations étaient tenus de signer un accord de confidentialité et de déclarer par écrit toutes situations de possible conflit d'intérêts direct ou indirect. Tous les conflits d'intérêts déclarés ont été analysés par le président du comité consultatif pour les Recommandations canadiennes pour les pratiques optimales de soins de l'AVC et par les membres concernés du personnel de Cœur + AVC pour déceler tout impact potentiel. Les candidats potentiels au comité de rédaction ayant déclaré des conflits d'intérêts directs n'ont pas été invités à participer.

Au début des discussions sur une thématique donnée, nous avons identifié les participants ayant des intérêts concurrents indirects en ce qui concerne cette thématique. Le comité de rédaction et les examinateurs externes travaillent dans des contextes médicaux très diversifiés. Cette approche interdisciplinaire a fait en sorte que les points de vue des diverses spécialités médicales et des différents milieux de soins concernés ont été pris en compte dans la préparation des recommandations; et aucun des membres n'a exercé plus d'influence qu'un autre, réduisant ainsi le risque d'intérêts concurrents potentiels ou réels chez les différents membres.

Étant donné l'absence d'intérêts concurrents directs relatifs à l'AAS, tous les membres ont pu prendre part au vote. Les 18 membres ont voté en faveur des recommandations pour la prévention secondaire et la prise de décision partagée, et 17 des 18 membres ont voté en faveur des recommandations pour la prévention primaire. Par conséquent, tout intérêt concurrent indirect potentiel s'est trouvé amenuisé par l'appui général de l'ensemble des membres du comité de rédaction. 


\section{Mise en œuvre}

Ces recommandations s'appuient sur les renseignements complémentaires présentés à l'adresse https://www.pratiquesoptimalesavc. ca/recommandations/AAS-par-prevention, notamment la justification de l'inclusion de cette thématique dans les Recommandations canadiennes pour les pratiques optimales de soins de l'AVC et une description du problème et du fardeau qu'elle représente; ses implications pour les systèmes chargés d'assurer l'accessibilité aux ressources et aux éléments structurels afférents aux niveaux de soins recommandés, y compris la formation professionnelle et la sensibilisation du public; les mesures de rendement après sa mise en œuvre; la prestation des soins et les résultats chez les patients; le déploiement de ressources pour les médecins et le public afin de promouvoir l'intégration des mesures d'intervention recommandées dans la pratique quotidienne; et un sommaire plus détaillé des données probantes sur lesquelles les recommandations ont été fondées. On peut aussi trouver des informations sur l'application des connaissances dans le site Web.

Une vaste gamme d'activités de sensibilisation des professionnels et du grand public sont prévues, notamment, la publication de messages dans les médias sociaux et traditionnels, des webinaires pour le public et les professionnels de la santé, des présentations lors de congrès, des campagnes par courriel et des avis dans les bulletins destinés aux professionnels de la santé et au public, une information à jour sur les sites Web de Cœur + AVC et des associations professionnelles qui ont appuyé les recommandations, et un document infographique pour expliquer celles-ci au public.

En ce qui concerne la pratique clinique, nous conseillons que les personnes qui prennent de l'AAS en prévention primaire soient informées des preuves les plus récentes selon lesquelles ce traitement conférerait moins de bienfaits qu'on l'a d'abord cru et que de nouvelles lignes directrices encadrent son utilisation. Les médecins sont fortement encouragés à modifier les traitements médicamenteux et à promouvoir l'adoption d'un meilleur mode de vie et d'habitudes plus saines, qui sont des mesures plus efficaces que l'AAS pour prévenir les événements cardiovasculaires; ces mesures comprennent la gestion de l'hypertension artérielle, du diabète et de l'hypercholestérolémie; l'abandon du tabagisme; l'augmentation de l'activité physique; le maintien d'un poids santé; et une alimentation saine ${ }^{34,35}$.

\section{Autres lignes directrices}

Ces lignes directrices se fondent sur les essais cliniques et revues systématiques les plus récents sur le sujet. Contrairement aux lignes directrices précédentes, nous ne recommandons pas l'AAS en prévention primaire d'un premier événement vasculaire pour aucun sous-groupe, même les patients exposés à un risque sur 10 ans estimé plus élevé. Les essais récents ne sont pas arrivés à démontrer hors de tout doute que l'AAS réduit le risque d'un premier événement vasculaire, et ils fournissent des preuves claires d'un risque accru de saignement majeur.

Les lignes directrices précédentes émises par la Société canadienne de cardiologie en 2011 stipulaient que les antiplaquettaires n'étaient pas recommandés chez les personnes sans maladie cardio- vasculaire en raison du risque accru de saignement majeur ${ }^{9}$. Les lignes directrices européennes de 2016 sur la prévention de la maladie cardiovasculaire ${ }^{36}$ incluent des recommandations similaires. En 2018, l'organisme Thrombose Canada suggérait de n'utiliser l'AAS que dans certains cas particuliers pour la prévention primaire de la morbidité et de la mortalité cardiovasculaires ${ }^{8}$.

Adoptées en 2019, les lignes directrices actuelles de l'American College of Cardiology/American Heart Association sur la prévention primaire de la maladie cardiovasculaire laissent entendre que l'AAS à faible dose (75-100 mg/j) pourrait être envisagé chez certains adultes de 40 à 70 ans exposés à un risque accru de maladie cardiovasculaire et serait à éviter chez les personnes de plus de 70 ans $^{37}$. Cela diffère de la recommandation de 2014, selon laquelle "l'utilisation de l'AAS pour la prophylaxie cardiovasculaire (y compris l'AVC, mais pas spécifique à cet événement vasculaire) est envisageable chez les personnes exposées à un risque suffisamment élevé (risque sur 10 ans > $10 \%$ ), car les bienfaits du traitement en surpassent les inconvénients $»^{38}$.

En 2016, le US Preventive Services Task Force a formulé des recommandations spécifiques à l'âge, selon lesquelles les personnes de 50 à 59 ans pourraient commencer à prendre de l'AAS à faible dose si leur risque cardiovasculaire sur 10 ans est supérieur à $10 \%$ et que leur risque de saignement n'est pas augmenté. Pour les personnes de 60 à 69 ans, la recommandation relevait d'une décision personnelle, moyennant un risque similaire sur 10 ans; selon le groupe de travail, les preuves ne permettaient pas de formuler des recommandations pour les personnes de moins de 50 ans et de plus de 69 ans $^{39}$. Le Groupe d'étude canadien sur les soins de santé préventifs n'a pas publié de recommandations à ce sujet. On trouve dans l'encadré no 3 des détails sur les lignes directrices passées en revue et analysées par le comité de rédaction.

\section{Lacunes dans les connaissances}

Il est probable que les données probantes récentes et la multiplication des lignes directrices selon lesquelles l'AAS n'est pas recommandé en prévention primaire de la maladie vasculaire entraîneront un changement substantiel des approches à la prévention primaire de la maladie vasculaire et de la perception du public; la pratique clinique s'en trouvera modifiée. Toutefois, d'importantes questions demeurent, sur lesquelles la recherche devra se pencher.

La plupart des essais ont porté sur les personnes âgées parce que celles-ci ont généralement un risque accru de nouveaux événements cardiovasculaires; on a émis une hypothèse, non confirmée, selon laquelle l'AAS pourrait procurer un bienfait réel chez des patients plus jeunes qui sont à risque très élevé, mais qui sont moins susceptibles de présenter des saignements digestifs. Compte tenu de la sensibilité croissante des techniques d'imagerie, il est désormais possible d'identifier les personnes présentant une maladie sous-clinique (par exemple, infarctus du myocarde ou AVC silencieux, ou athérosclérose asymptomatique), mais on ignore si elles peuvent tirer profit d'un traitement avec l'AAS. Les essais disponibles auprès de ce type de population n'ont pas montré que l'AAS modifie de manière cliniquement substantielle le risque d'un tout premier événement clinique ${ }^{14,15}$. 


\section{Organisme (année)}

\section{Recommandation*}

American Diabetes Association (2019) ${ }^{40}$

ACC/AHA Guideline on the Primary Prevention of Cardiovascular Disease $(2019)^{37}$

Thrombose Canada (2018)

$\operatorname{USPSTF}(2016)^{39}$

Sixth Joint Task Force of the European Society of Cardiology and Other Societies on Cardiovascular Disease Prevention in Clinical Practice (2016) $)^{36}$

American College of Chest Physicians $(2012)^{41}$

Société canadienne de cardiologie $(2011)^{9}$ niveau de preuve A). catégorie III, niveau de preuve B-R). Thrombose Canada.) de 50 ans (énoncé I). ou plus (énoncé I). preuve $2 \mathrm{~B})$.
- Le traitement par aspirine (75-162 mg par jour) peut être envisagé comme stratégie de prévention primaire chez les patients atteints de diabète exposés à un risque accru de MCV, après discussion avec le patient au sujet des bienfaits c. l'accroissement du risque de saignement (niveau de preuve C).

- L'aspirine à faible dose (75-100 mg, PO/jour) peut être envisagée en prévention primaire de la MCV athéroscléreuse chez certains adultes de 40 à 70 ans exposés à un risque élevé de MCV athéroscléreuse, mais non à un risque accru de saignement (recommandation de catégorie Ilb,

- L'aspirine à faible dose (75-100 mg PO/jour) ne doit pas être administrée d'emblée pour la prévention primaire de la MCV athéroscléreuse chez les adultes $>70$ ans (recommandation de

- L'aspirine à faible dose (75-100 mg PO/jour) ne doit pas être administrée en prévention primaire de la MCV athéroscléreuse chez des adultes de tout âge exposés à un risque accru de saignement (recommandation de catégorie III, niveau de preuve C-LD).

- Seulement dans certains cas particuliers, chez des patients sans maladie vasculaire évidente ayant un risque vasculaire élevé et un risque faible de saignement. Exemples : athérosclérose carotidienne ou coronarienne asymptomatique confirmée aux épreuves d'imagerie vasculaire et patients exposés à un risque très élevé d'événement vasculaire en raison de multiples facteurs de risque cardiovasculaire. (Aucun niveau de preuve fourni dans les énoncés de l'organisme

- L'USPSTF recommande l'instauration de l'aspirine à faible dose pour la prévention primaire de la MCV et du CCR chez les adultes de 50 à 59 ans exposés à un risque de MCV de $10 \%$ ou plus sur 10 ans, qui ne sont pas exposés à un risque accru de saignement, qui ont une espérance de vie d'au moins 10 ans et qui acceptent de prendre quotidiennement de l'aspirine à faible dose pendant au moins 10 ans (recommandation de niveau B).

- La décision d'instaurer l'aspirine à faible dose en prévention primaire de la MCV et du CCR chez les adultes de 60 à 69 ans exposés à un risque de MCV de $10 \%$ ou plus sur 10 ans doit être évaluée au cas par cas. Les personnes qui ne sont pas exposées à un risque accru de saignement, qui ont une espérance de vie d'au moins 10 ans et qui acceptent de prendre quotidiennement de l'aspirine à faible dose pendant au moins 10 ans sont plus susceptibles d'en tirer des bienfaits. Les personnes qui accordent plus d'importance aux bienfaits potentiels qu'aux risques éventuels peuvent choisir de commencer à prendre de l'aspirine à faible dose (recommandation de catégorie C).

- Les preuves actuelles sont insuffisantes pour établir les bienfaits et les risques associés à l'instauration de l'aspirine en prévention primaire de la MCV et du CCR chez les adultes de moins

- Les preuves actuelles sont insuffisantes pour établir les bienfaits et les risques associés à l'instauration de l'aspirine en prévention primaire de la MCV et du CCR chez les adultes de 70 ans

- Le traitement antiplaquettaire n'est pas recommandé chez les personnes sans MCV en raison d'un risque accru de saignement majeur (recommandation de catégorie III; niveau de preuve B).

- Pour les personnes de 50 ans ou plus, sans AVC symptomatique, nous suggérons l'aspirine à faible dose (75 à $100 \mathrm{mg}$ par jour) plutôt que l'absence de traitement par aspirine (niveau de

- Chez les hommes et les femmes ne présentant aucun signe évident de maladie vasculaire, l'utilisation d'AAS, peu importe la dose, n'est pas recommandée d'emblée en prévention des accidents vasculaires ischémiques (recommandation de catégorie III; niveau de preuve A).

- Chez les hommes et les femmes ne présentant aucun signe évident de maladie vasculaire, l'utilisation de clopidogrel $75 \mathrm{mg}$ par jour plus AAS, peu importe la dose, n'est pas recommandée en prévention des accidents vasculaires ischémiques (recommandation de catégorie III; niveau de preuve B).

- Dans certains cas particuliers, chez les hommes et les femmes ne présentant aucun signe évident de maladie vasculaire et ayant un risque élevé de problèmes vasculaires et un risque faible de saignement, l'AAS à 75-162 mg par jour peut être envisagé (recommandation de catégorie llb; niveau de preuve $\mathrm{C}$ ).

Note: $\mathrm{ACA} / \mathrm{AHA}=$ American College of Cardiology/American Heart Association, $\mathrm{AAS}=$ acide acétylsalicylique, $\mathrm{CCR}=$ cancer colorectal, $\mathrm{MCV}=$ maladie cardiovasculaire, USPSTF = US

*Les définitions des catégories ou de la solidité des recommandations et des niveaux de preuve se trouvent dans les lignes directrices citées. 
En terminant, il reste la question délicate des conseils à donner aux patients qui prennent de l'AAS en prévention primaire depuis de nombreuses années sans présenter d'effets indésirables comme les saignements digestifs. On dispose de peu de recherches sur l'effet de la cessation de la prise quotidienne d'AAS dans ce contexte, et aucun essai contrôlé n'a été réalisé. La cessation de l'AAS n'est peut-être pas à conseiller chez tous les patients s'il risque de provoquer le retour des effets prothrombotiques. En outre, la documentation sur la prise de décision partagée n'aborde pas cette thématique précise, ce qui rappelle que l'occasion est là d'appliquer les principes de la prise de décision partagée à ce sujet qui touche probablement un nombre substantiel de personnes au pays.

\section{Conclusion}

L'utilisation de l'AAS pour la prévention (primaire et secondaire) des événements vasculaires est une pratique répandue au Canada et ailleurs dans le monde depuis des décennies. À la lumière d'une réévaluation des données probantes et des publications récentes émanant de volumineux essais impartiaux, nous recommandons aujourd'hui de ne pas utiliser d'emblée l'AAS en prévention primaire chez la plupart des gens. Il faut informer la population que la prise quotidienne d'AAS n'est pas recommandée en prévention primaire des événements vasculaires, car les risques de saignement pourraient dépasser les bienfaits escomptés du traitement.

L'AAS est encore fortement recommandé en prévention secondaire chez les patients qui ont présenté une maladie cardiovasculaire, vasculaire cérébrale ou artérielle périphérique évidente.

La décision d'instaurer, de cesser ou de maintenir un traitement avec l'AAS est individualisée et le processus de prise de décision doit être partagé entre les professionnels de la santé et les patients, après analyse des risques, bienfaits, valeurs et préférences.

En terminant, les médecins sont fortement encouragés à modifier les traitements médicamenteux et à promouvoir l'adoption d'un meilleur mode de vie et d'habitudes plus saines, car ce sont des mesures plus efficaces que l'AAS pour prévenir les événements cardiovasculaires.

Ces recommandations ont pour but de modifier les pratiques, d'accroître la sensibilisation et de susciter des changements à l'échelle des politiques et des systèmes, en plus d'améliorer la qualité des soins pour prévenir les événements vasculaires, tout en soulignant l'importance de veiller à des environnements favorables et d'appliquer des stratégies efficaces en prévention secondaire.

\section{Références}

1. (Dis)connected: how unseen links are putting us at risk - 2019 report on heart, stroke and vascular cognitive impairment. Accessible ici : www.heartandstroke.ca/-/ media/pdf-files/canada/2019-report/heartandstrokereport2019.ashx (consulté le 22 novembre 2019).

2. Gaziano JM, Brotons C, Coppolecchia R, et al.; ARRIVE Executive Committee. Use of aspirin to reduce risk of initial vascular events in patients at moderate risk of cardiovascular disease (ARRIVE): a randomised, double-blind, placebocontrolled trial. Lancet 2018;392:1036-46.

3. McNeil JJ, Nelson MR, Woods RL, et al.; ASPREE Investigator Group. Effect of aspirin on all-cause mortality in the healthy elderly. N Engl J Med 2018;379:1519-28.

4. ASCEND Study Collaborative Group; Bowman L, Mafham M, Wallendszus K, et al. Effects of aspirin for primary prevention in persons with diabetes mellitus. NEngl J Med 2018;379:1529-39.
5. Zheng SL, Roddick AJ. Association of aspirin use for primary prevention with cardiovascular events and bleeding events: a systematic review and metaanalysis. JAMA 2019;321:277-87.

6. Mahmoud AN, Gad MM, Elgendy AY, et al. Efficacy and safety of aspirin for primary prevention of cardiovascular events: a meta-analysis and trial sequential analysis of randomized controlled trials. Eur Heart J 2019;40:607-17.

7. O'Brien CW, Juraschek SP, Wee CC. Prevalence of aspirin use for primary prevention of cardiovascular disease in the United States: results from the 2017 National Health Interview Survey. Ann Intern Med 201923 juillet [Cyberpublication avant impression]. doi: 10.7326/M19-0953.

8. Acetylsalicylic acid (ASA). Whitby (ON): Thrombosis Canada; 2018. Accessible ici : https://thrombosiscanada.ca/wp-content/uploads/2019/01/Acetylsalicyclic -Acid-2018July24-Final.pdf (consulté le 22 novembre 2019).

9. Bell AD, Roussin A, Cartier R, et al.; Canadian Cardiovascular Society. The use of antiplatelet therapy in the outpatient setting: Canadian Cardiovascular Society guidelines. Can J Cardiol 2011;27(Suppl A):S1-59.

10. Wein T, Lindsay MP, Côté R, et al.; Heart and Stroke Foundation Canadian Stroke Best Practice Committees. Canadian stroke best practice recommendations: secondary prevention of stroke, sixth edition practice guidelines, update 2017. Int J Stroke 2018;13:420-43.

11. Antithrombotic Trialists' Collaboration. Collaborative meta-analysis of randomised trials of antiplatelet therapy for prevention of death, myocardial infarction, and stroke in high risk patients. BMJ 2002;324:71-86.

12. Antithrombotic Trialists' (ATT) Collaboration; Baigent C, Blackwell L, Collins R, et al. Aspirin in the primary and secondary prevention of vascular disease: collaborative meta-analysis of individual participant data from randomised trials. Lancet 2009;373:1849-60.

13. Berger JS, Brown DL, Becker RC. Low-dose aspirin in patients with stable cardiovascular disease: a meta-analysis. Am J Med 2008;121:43-9.

14. Fowkes FG, Price JF, Stewart MC, et al.; Aspirin for Asymptomatic Atherosclerosis Trialists. Aspirin for prevention of cardiovascular events in a general population screened for a low ankle brachial index: a randomized controlled trial. JAMA 2010;303:841-8.

15. Côté R, Battista RN, Abrahamowicz M, et al. Lack of effect of aspirin in asymptomatic patients with carotid bruits and substantial carotid narrowing. The Asymptomatic Cervical Bruit Study Group. Ann Intern Med 1995;123:649-55.

16. Sepucha KR, Scholl I. Measuring shared decision making: a review of constructs, measures, and opportunities for cardiovascular care. Circ Cardiovasc Qual Outcomes 2014;7:620-6.

17. Alonso-Coello P, Montori VM, Díaz MG, et al. Values and preferences for oral antithrombotic therapy in patients with atrial fibrillation: physician and patient perspectives. Health Expect 2015;18:2318-27.

18. Belch J, MacCuish A, Campbell I, et al.; Prevention of Progression of Arterial Disease and Diabetes Study Group; Diabetes Registry Group; Royal College of Physicians Edinburgh. The prevention of progression of arterial disease and diabetes (POPADAD) trial: factorial randomised placebo controlled trial of aspirin and antioxidants in patients with diabetes and asymptomatic peripheral arterial disease. BMJ 2008;337:a1840.

19. Huang WY, Saver JL, Wu YL, et al. Frequency of intracranial hemorrhage with low-dose aspirin in individuals without symptomatic cardiovascular disease: a systematic review and meta-analysis. JAMA Neurol 2019 May 13 [Cyberpublication avant impression]. doi: 10.1001/jamaneurol.2019.1120.

20. Marquis-Gravel G, Roe MT, Harrington RA, et al. Revisiting the role of aspirin for the primary prevention of cardiovascular disease. Circulation 2019;140: 1115-24.

21. Rothwell PM, Cook NR, Gaziano JM, et al. Effects of aspirin on risks of vascular events and cancer according to bodyweight and dose: analysis of individual patient data from randomised trials. Lancet 2018;392:387-99.

22. Berger JS, Roncaglioni MC, Avanzini F, et al. Aspirin for the primary prevention of cardiovascular events in women and men: a sex-specific meta-analysis of randomized controlled trials. JAMA 2006;295:306-13.

23. Charles C, Gafni A, Whelan T. Shared decision-making in the medical encounter: what does it mean? (or it takes at least two to tango). Soc Sci Med 1997;44:681-92.

24. Grad R, Légaré $F$, Bell NR, et al. Shared decision making in preventive health care: what it is; what it is not. Can Fam Physician 2017;63:682-4.

25. Johnson RA, Huntley A, Hughes RA, et al. Interventions to support shared decision making for hypertension: a systematic review of controlled studies. Health Expect 2018;21:1191-207.

26. Tinsel I, Buchholz A, Vach W, et al. Shared decision-making in antihypertensive therapy: a cluster randomised controlled trial. BMC Fam Pract 2013; 14:135.

27. Loewen PS, Bansback N, Hicklin J, et al. Evaluating the effect of a patient decision aid for atrial fibrillation stroke prevention therapy. Ann Pharmacother 2019;53:665-74. 
28. Bailey RA, Pfeifer M, Shillington AC, et al. Effect of a patient decision aid (PDA) for type 2 diabetes on knowledge, decisional self-efficacy, and decisional conflict. BMC Health Serv Res 2016;16:10.

29. Harris J, Teed M, Gierman N, et al. Bringing the patient voice to life in stroke best practice guidelines [abstract]. Int J Stroke 2018;13:102. doi: 10.1177 /1747493018789543.

30. Graham ID, Harrison MB, Brouwers M, et al. Facilitating the use of evidence in practice: evaluating and adapting clinical practice guidelines for local use by health care organizations. J Obstet Gynecol Neonatal Nurs 2002;31:599-611.

31. Vernooij RW, Alonso-Coello P, Brouwers M, et al.; CheckUp Panel. Reporting items for updated clinical guidelines: checklist for the reporting of updated guidelines (CheckUp). PLoS Med 2017;14:e1002207.

32. Brouwers MC, Kho ME, Browman GP, et al.; AGREE Next Steps Consortium. AGREE II: advancing guideline development, reporting and evaluation in health care. CMAJ 2010;182:E839-42.

33. Guyatt GH, Cook DJ, Jaeschke R, et al. Grades of recommendation for antithrombotic agents: American College of Chest Physicians Evidence-Based Clinical Practice Guidelines (8th Edition). Chest 2008;133(Suppl): 123S-31S.

34. Yusuf S, Hawken S, Ounpuu S, et al.; INTERHEART Study Investigators. Effect of potentially modifiable risk factors associated with myocardial infarction in 52 countries (the INTERHEART study): case-control study. Lancet 2004;364: 937-52.

35. O'Donnell MJ, Chin SL, Rangarajan S, et al.; INTERSTROKE investigators. Global and regional effects of potentially modifiable risk factors associated with acute stroke in 32 countries (INTERSTROKE): a case-control study. Lancet 2016;388:761-75.
36. Piepoli MF, Hoes AW, Agewall S, et al.; ESC Scientific Document Group. 2016 European Guidelines on cardiovascular disease prevention in clinical practice: The Sixth Joint Task Force of the European Society of Cardiology and Other Societies on Cardiovascular Disease Prevention in Clinical Practice (constituted by representatives of 10 societies and by invited experts) Developed with the special contribution of the European Association for Cardiovascular Prevention \& Rehabilitation (EACPR). Eur Heart J 2016;37:2315-81.

37. Arnett DK, Blumenthal RS, Albert MA, et al. 2019 ACC/AHA Guideline on the Primary Prevention of Cardiovascular Disease: a report of the American College of Cardiology/American Heart Association Task Force on Clinical Practice Guidelines. Circulation 2019;140:e596-646.

38. Meschia JF, Bushnell C, Boden-Albala B, et al.; American Heart Association Stroke Council; Council on Cardiovascular and Stroke Nursing; Council on Clinical Cardiology; Council on Functional Genomics and Translational Biology; Council on Hypertension. Guidelines for the primary prevention of stroke: a statement for healthcare professionals from the American Heart Association/ American Stroke Association. Stroke 2014;45:3754-832.

39. Bibbins-Domingo K; U.S. Preventive Services Task Force. Aspirin use for the primary prevention of cardiovascular disease and colorectal cancer: U.S. Preventive Services Task Force recommendation statement. Ann Intern Med 2016;164:836-45.

40. American Diabetes Association. 10. Cardiovascular disease and risk management: Standards of Medical Care in Diabetes - 2019. Diabetes Care 2019;42:S103-23.

41. Vandvik PO, Lincoff AM, Gore JM, et al. Primary and secondary prevention of cardiovascular disease: Antithrombotic Therapy and Prevention of Thrombosis, 9th ed: American College of Chest Physicians Evidence-Based Clinical Practice Guidelines. Chest 2012;141:e637S-68S.
Auteurs : Theodore Wein MD, M. Patrice Lindsay inf. PhD, David G. Gladstone M. PhD, Alexandre Poppe MDCM, Alan Bell MD, Leanne K. Casaubon MD MSc, Norine Foley MSc, Shelagh B. Coutts MD MBChB, Jafna Cox MD, James Douketis MD, Thalia Field MD MScS, Laura Gioia MD MSc, Jeffrey Habert MD, Eddy Lang MD MDCM, Shamir R. Mehta MD MSc, Christine Papoushek PharmD, William Semchuk PharmD MSc, Mikul Sharma MD MSc, Jacob A. Udell MD MSP, Stephanie Lawrence BJ, Anita Mountain MD, Gord Gubitz MD, Dar Dowlatshahi MD PhD, Anne Simard MScS BJ, Andrea de Jong inf. MN, Eric E. Smith MD MSP, pour la Fondation des maladies du cœur et de l'AVC du Canada en collaboration avec le Consortium Neurovasculaire Canadien.

Intérêts concurrents : Theodore Wein déclare avoir reçu des subventions d'Allergan, de Boehringer Ingelheim et de Bayer, et des subventions et honoraires de consultant de Servier, d'Allergan et d'Ipsen; il a offert des séances de formation médicale continue pour Servier; et il a reçu un remboursement de frais de déplacement de Servier. David Gladstone n'a eu aucun lien personnel de nature financière avec l'industrie au cours des 36 mois précédant la publication. Il agit à titre d'investigateur principal de l'étude SCREEN-AF (sans rémunération; étude soutenue par le Réseau canadien pour la prévention des accidents cérébrovasculaires [C-SPIN], et subventionnée par les Instituts de recherche en santé du Canada [IRSC]), et à titre de co-investigateur principal pour le volet canadien de l'étude ARCADIA, subventionnée par les National Institutes of Health des États-Unis. Le $D^{r}$ Gladstone a déjà été superviseur indépendant de l'innocuité pour cet essai (sans rémunération); investigateur principal d'un projet de recherche du réseau C-SPIN subventionné par les IRSC pour la base de données Ontario Holter/Echo; et investigateur d'un site local des études NAVIGATE ESUS et NASPAF-ICH (tous honoraires des sites versés à l'établissement). Alexandre Poppe est investigateur principal et coinvestigateur de sites pour NoNo et Bayer et a reçu une bourse de l'initiative Canadian Stroke Trials for Optimized Results. Alan Bell déclare avoir reçu des honoraires personnels d'AstraZeneca Novartis, de Pfizer, de Servier et de Bayer, en dehors du travail soumis. Il est viceprésident de l'organisme Thrombose Canada (poste non rémunéré), il fait partie du conseil d'administration d'Hypertension Canada (poste non rémunéré) et il est l'auteur des Lignes directrices canadiennes relatives au traitement antiplaquettaire de la Société canadienne de cardiologie. Leanne K. Casaubon déclare avoir reçu des honoraires personnels de Medtronic et de Bayer et est investigatrice principale de site pour un essai clinique relatif au NA1 de NoNo Inc. (aucune compensation financière personnelle). James Douketis déclare avoir reçu des honoraires personnels de Leo Pharma, de Janssen, de Pfizer, de BristolMyers Squibb, de Sanofi, de Servier Canada et de Portola, lesquels sont déposés dans des comptes de recherche en milieu hospitalier (St. Joseph's Healthcare Hamilton) et universitaire (Université McMaster) ou d'organismes de bienfaisance. Le Dr Douketis déclare aussi avoir reçu des honoraires personnels en tant qu'employé de The Merck Manual et d'UpToDate. Thalia Field déclare avoir reçu des subventions et des honoraires personnels de Bayer Canada, et des honoraires personnels de Pfizer-BMS et de Servier, en dehors du travail soumis. Jeffrey Habert déclare avoir reçu des honoraires personnels de Pfizer, d'Amgen, de BMS, de Bayer, de Boehringer Ingelheim, d'Eli-Lilly, de Purdue, d'Allergan, d'AstraZeneca, de Lundbeck, de Novo-Nordisk, de Servier, de Janssen, de Roche, de HLS, d'Otsuka (conférencier, membre de comité consultatif ou consultant), et d'Alliance, de M.D.Briefcase, de Bausch, de Liv, de MedPlan, de Brandaide, d'Academy, de Bridge, de Seacourses, de Meducom, du Centre International de développement professionnel en santé et médecine, de CPD Network, d'Antibody, de CHRC, de STA Healthcare Communications, de CTC, du Canadian Collaborative Research Network, de Four Health Comm (membre de comité scientifique et consultant), en dehors du travail soumis. Shamir Mehta déclare avoir reçu des subventions et des honoraires personnels d'AstraZeneca, des subventions de Boston Scientific et des honoraires personnels de Bayer. William Semchuk déclare avoir reçu des honoraires personnels de Bayer, de Pfizer, de Servier, de BMS, de Sanofi et d'AstraZeneca, en dehors du travail soumis. Mikul Sharma déclare avoir reçu des subventions et des honoraires de Bayer, des subventions de Boehringer Ingelheim et de Bristol-Myers Squibb, et des honoraires de consultant de Portola et de Daiichi Sankyo. Jacob Udell déclare avoir reçu des subventions et des honoraires personnels d'AstraZeneca, de Boehringer Ingelheim, de Janssen et de Sanofi. Eric E. Smith a reçu des honoraires personnels de Portola Pharmaceuticals et de Biogen. Dar Dowlatshahi a reçu une bourse de la Fondation des maladies du cœur et de l'AVC du Canada et des honoraires de Bayer, de BMS et d'Apopharma, en dehors du travail soumis. Gord Gubitz est membre de comités consultatifs pour Bayer, Boehringer Ingelheim et Pfizer. Aucun autre intérêt concurrent n'a été déclaré.

Cet article a fait l'objet d'un examen par les pairs. 
Affiliations : Département de neurologie et neurochirurgie [Wein], Université McGill, Montréal (Qc); Fondation des maladies du cœur et de l'AVC du Canada [Lindsay, Lawrence, Simard, de Jong]; Division de neurologie [Gladstone, Casaubon], Département de médecine, Université de Toronto; Division de neurologie [Gladstone], Service de médecine, Centre régional de traitement des AVC; Programme de sciences neurologiques Hurvitz [Gladstone], Centre des sciences de la santé Sunnybrook; Institut de recherche Sunnybrook [Gladstone], Toronto (Ont.); Centre hospitalier de l'Université de Montréal (CHUM) [Poppe, Gioia], Hôpital Notre-Dame, Montréal (Qc); Département de médecine familiale [Bell, Habert], Université de Toronto; Programme de traitement des AVC de l'hôpital Toronto Western [Casaubon], Réseau universitaire de santé, Toronto (Ont.); workHORSE Consulting Ltd. [Foley], London (Ont.); Département de neurosciences cliniques [Coutts, Smith], École de médecine Cumming, Université de Calgary, Calgary (Alb.); Faculté de médecine (cardiologie) [Cox], Université Dalhousie, Halifax (N.-É.); Département de médecine [Douketis], Université McMaster, Hamilton (Ont.); Division de neurologie [Field], Département de médecine, Université de la Colombie-Britannique, Vancouver (C.-B.); Département de médecine d'urgence [Lang], École de médecine Cumming, Université de Calgary, Calgary Alb.); Division de cardiologie [Mehta], Département de médecine, Université McMaster, Hamilton (Ont.); Département de médecine familiale et communautaire [Papoushek], Faculté de pharmacie Leslie-Dan, Université de Toronto, Toronto (Ont.); École de pharmacie [Semchuk], Université de la Saskatchewan, Saskatoon (Sask.); Division de neurologie [Sharma], Département de médecine, Université McMaster, Hamilton (Ont.); Division cardiovasculaire [Udell], Service de médecine, Hôpital Women's College; Centre de cardiologie Peter-Munk [Udell], Hôpital général de Toronto, Université de Toronto, Toronto (Ont.); Divisions de physiatrie et réadaptation [Mountain] et de neurologie [Gubitz], Département de médecine, Université Dalhousie; Division de neurologie [Dowlatshahi], Faculté de médecine, Université d'Ottawa, Ottawa (Ont.).

Collaborateurs : Theodore Wein est l'auteur principal et le président du comité d'experts chargé de la rédaction de L'acide acétylsalicylique (AAS) pour la prévention des accidents vasculaires; il a participé à tous les aspects de la conception et de l'élaboration du travail, à la collecte, à l'analyse et à l'interprétation des données, et à la rédaction de l'ébauche des recommandations et du présent manuscrit. M. Patrice Lindsay est l'auteure/la personne-ressource, elle fait partie des rédacteurs principaux des lignes directrices des Recommandations canadiennes pour les pratiques optimales de soins de l'AVC et du présent manuscrit; elle a participé à tous les aspects de la revue de la documentation scientifique, aux délibérations du comité de rédaction, au processus de révision externe et à la préparation du manuscrit; elle a rédigé des documents complémentaires. Eric Smith est l'un des rédacteurs principaux de ce manuscrit et est co-président du comité consultatif sur les Pratiques optimales de soins de l'AVC au Canada, il a participé à tous les aspects de la conception et de l'élaboration du travail, à la collecte, à l'analyse et à l'interprétation des données, et à la rédaction de l'ébauche des recommandations et du présent manuscrit. Norine Foley a procédé aux interrogations sur les données probantes et a créé les tableaux afférents soumis au comité de rédaction en plus de participer à tous les aspects de la rédaction de l'ébauche du manuscrit. David Gladstone, Alexandre Poppe, Alan Bell, Leanne Casaubon, Shelagh Coutts, Jafna Cox, James Douketis, Thalia Field, Laura Gioia, Jeffrey Habert, Eddy Lang, Shamir Mehta, Christine Papoushek, William Semchuk, Mikul Sharma, Jacob Udell, Andrea de Jong et Anne Simard ont activement participé au comité de rédaction formé d'experts chargés d'analyser les données probantes, de réviser les versions successives de l'ébauche et de dresser la liste de recommandations finale. Anita Mountain, Gord Gubitz et Dariush Dowlatshahi sont des membres chevronnés du comité consultatif sur les Pratiques optimales de soins de l'AVC du Canada et ont commenté chaque étape de la conception de ces recommandations et révisé les versions successives de l'ébauche du manuscrit soumis pour approbation. Stephanie Lawrence a contribué à l'ébauche du manuscrit et aux communications afférentes, ainsi qu'à la documentation relative à l'application des connaissances. Tous les auteurs ont donné leur approbation finale pour la version soumise pour publication et assument l'entière responsabilité de tous les aspects du travail.

Financement : La préparation des Recommandations canadiennes pour les pratiques optimales de soins de l'AVC est entièrement financée par la Fondation des maladies du cœur et de l'AVC du Canada. La préparation de ces lignes directrices n'a bénéficié du financement d'aucune entité commerciale, y compris des fabricants de produits pharmaceutiques ou de dispositifs médicaux. Les membres des comités de rédaction des recommandations et les examinateurs externes ont travaillé bénévolement et n'ont reçu aucune rémunération pour leur participation à la rédaction, aux mises à jour et aux révisions des lignes directrices.

Appui : Ces lignes directrices sont appuyées par le Consortium Neurovasculaire Canadien, la Société canadienne de cardiologie, l'organisme Thrombose Canada, la Société canadienne des pharmaciens d'hôpitaux, l'Association des pharmaciens du Canada et la Nurse Practitioners' Association of Ontario.

Remerciements : La Fondation des maladies du cœur et de l'AVC du Canada remercie sincèrement les responsables et les membres du comité de rédaction du document L'AAS pour la prévention primaire des accidents vasculaires, lesquels ont tous consacré gratuitement de leur temps et leur expertise pour mettre ces recommandations à jour. La fondation remercie spécialement les membres du comité communautaire de consultation et révision, lesquels ont relu toutes les sections de ce module et partagé leurs expériences et réflexions personnelles sur ce qui a rendu ou aurait dû rendre leur mandat optimal; il s'agit de Cheryl Beattie, de Jennifer Bogart, de Dan Dobbin, de Glen Hilton, de Judy Hilton, d'Allan Morrison et d'Anjie Valgardson. Des membres du Consortium Neurovasculaire Canadien, de la Société canadienne de cardiologie, de l'organisme Thrombose Canada et de la Société canadienne des pharmaciens d'hôpitaux ont participé à la préparation de ces recommandations. La Fondation des maladies du cœur et de l'AVC du Canada remercie l'Association des pharmaciens du Canada d'avoir relu l'ébauche des lignes directrices. Les recommandations ont fait l'objet d'un examen externe par Lana Castellucci, Robert Cote, Kim Connelly, John Eikelboom, Michael E. Farkouh, Karen Harkness, Robert Hegele, Andrew Krahn, Gabriela Lewin, Louise McCullough, Kara Nerenberg, Wieslaw Oczkowski, Stephen Phillips, Heather Purvis, Elissa Weinberg et Heather Williams. La fondation exprime sa gratitude à l'endroit du comité consultatif sur les pratiques optimales et la qualité des soins de l'AVC, notamment Eric Smith, Anita Mountain, Leanne K. Casaubon, Gord Gubitz, Dar Dowlatshahi, Dylan Blacquiere, Thalia Field, Louise Clément, Farrell Leibovitch, Christine Papoushek, Jeffrey Habert, Barbara Campbell, Joyce Fung, Michael Hill, Tim Hillier, Eddy Lang, Pascale Lavoie, Beth Linkewich, Colleen O'Connell, Melanie Penn, Jai Shankar, Debbie Timpson, Theodore Wein et Katie White, pour l'examen interne et les conseils tout au long du processus. La Fondation des maladies du cœur et de l'AVC du Canada remercie Norine Foley et l'équipe d'analyse des données probantes de workHORSE; Colleen Norris pour sa contribution aux enjeux relatifs au sexe et au genre dans les données probantes disponibles; Laurie Charest, de la fondation, pour la coordination des équipes chargées des Recommandations canadiennes pour les pratiques optimales de soins de l'AVC; et ses propres équipes internes qui ont contribué à la préparation et à la publication des recommandations : Communications, Services créatifs, Services linguistiques, Promotion du rétablissement, Politiques de santé et Solutions numériques.

Correspondance : M. Patrice Lindsay, Patrice.Lindsay@heartandstroke.ca 